\title{
Epigenetic regulation of CD271, a potential cancer stem cell marker associated with chemoresistance and metastatic capacity
}

\author{
SULAN LI ${ }^{1,2^{*}}$, DONGLI YUE ${ }^{1,3^{*}}$, XINFENG CHEN $^{1,3}$, LIPING WANG $^{3}$, JIEYAO LI $^{1,3}$, YU PING $^{1,4}$,

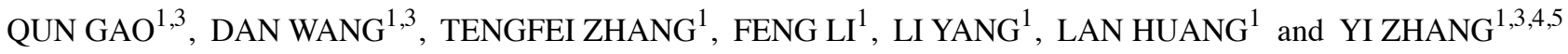 \\ ${ }^{1}$ Biotherapy Center, Departments of ${ }^{2}$ Radiology and ${ }^{3}$ Oncology, The First Affiliated Hospital of \\ Zhengzhou University; ${ }^{4}$ School of Life Sciences, Zhengzhou University; ${ }^{5}$ Institute of Clinical-Medicine, \\ The First Affiliated Hospital of Zhengzhou University, Zhengzhou, Henan 450052, P.R. China
}

Received August 19, 2014; Accepted October 3, 2014

DOI: $10.3892 /$ or.2014.3569

\begin{abstract}
Cancer stem cells (CSCs) are considered to be the cause of tumor initiation, metastasis and recurrence. Additionally, CSCs are responsible for the failure of chemotherapy and radiotherapy. The isolation and identification of CSCs is crucial for facilitating the monitoring, therapy or prevention of cancer. We aimed to identify esophageal squamous cell carcinoma (ESCC) stem-like cells, the epigenetic mechanism and identify novel biomarkers for targeting ESCC CSCs. Sixty-three paired ESCC tissues and adjacent noncancerous tissues were included in this study. CD271, which was identified as the CSC marker for melanoma, was assessed using quantitative PCR (qPCR). Using flow cytometry, we isolated CD271 ${ }^{+}$cells comprising $7.5 \%$ of cancer cells from the KYSE70 cell line. Sphere formation and anchorage-independent growth were analyzed in $\mathrm{CD} 271^{+}$and CD271- cancer cells, respectively. qPCR was used to detect stem-related genes and CCK-8 was performed to analyze the sensitivity to chemotherapy in the two groups. Bisulfite genomic sequencing was used to analyze the methylation status. CD271 expression was significantly higher in ESCC tissues than in adjacent non-cancerous tissues. Compared with CD271' cancer cells, CD $271^{+}$cancer cells showed a higher ability of sphere and colony formation, a high level expression of stem-related gene, and resistance to chemotherapy. The expression of CD271 was induced by a demethylation agent. In conclusion, CD271 ${ }^{+}$ ESCC cells possess stem-like properties. CD271 can potentially act as a prognostic marker for ESCC, whose expression is regulated epigenetically.
\end{abstract}

Correspondence to: Professor Yi Zhang, Biotherapy Center, The First Affiliated Hospital of Zhengzhou University, 1 Jianshe Road, Zhengzhou, Henan 450052, P.R. China

E-mail: yizhang@zzu.edu.cn

${ }^{*}$ Contributed equally

Key words: esophageal squamous cell carcinoma, cancer stem cell, CD271, DNA methylation

\section{Introduction}

Esophageal squamous cell carcinoma (ESCC) is a lethal malignancy with a 5-year survival rate of $26.2 \%$ due to late diagnosis, rapid growth and metastasis (1). Thus, it is necessary to identify new effective therapeutic strategies for ESCC, especially molecularly targeted therapies, based on a better understanding of the biological events of ESCC cells. Cancer stem cells (CSCs) are a limited number of cancer cells with a self-renewal potential and extensive proliferation capacity and play a dominant role in tumor initiation, metastasis and recurrence (2-4). In pancreatic cancer, a subpopulation of migrating $\mathrm{CD}_{133}{ }^{+} \mathrm{CXCR} 4{ }^{+} \mathrm{CSC}$ was reported to be essential for tumor metastasis (5). In glioma, CD133 ${ }^{+}$CSCs were associated with radioresistance and contributed to tumor recurrence after radiotherapy due to preferential activating DNA damage checkpoint response. In malignant melanoma, the drug transporter and chemoresistance mediator ABCB5 was identified as a novel molecular marker for a distinct subset of chemoresistant, stem-cell phenotype-expressing tumor cells, indicating that ABCB5 may be a specific target to enhance cytotoxic efficacy (6). Zhang et al also provided evidence that CSC-like cells may play a role in the progression and drug resistance of bladder cancer (7). The aforementioned studies showed that CSCs can be a good therapeutic target for various types of cancer. Although CSCs are common many types of tumors (8-11), the data for CSCs from human ESCC are conflicting.

CD271, a member of the tumor necrosis factor receptor superfamily (12), plays a role in cell proliferation, survival, and apoptosis (13). It is also known as a nerve growth factor receptor and interacts with neurotrophins (14). CD $271^{+}$cells have been reported to be genuine CSCs in human melanoma (15). CD271 ${ }^{+}$cells showed higher tumorigenecity and metastatic ability in melanoma (16). The high level of CD271 expression was correlated with a poor prognosis for patients with hypopharyngeal cancer (17). Okumura et al suggested CD271 as the human esophageal keratinocyte stem cell marker, which may be valuable for prospectively investigating stem cell regulation in association with different biological processes including the neoplastic transformation of regenerative epithelia (18). Authors of that study also identified CD271 
as being expressed in $49.2 \%$ ESCC and necessary for survival and maintenance of ESCC tumors (19). Huang et al demonstrated that $\mathrm{CD} 271^{+}$cells possess some characteristics of CSCs (20). However, there is no report with regard to the epigenetic regulation on $\mathrm{CD} 271$ expression.

In the present study, we confirmed that CD271+ ESCC cells exhibited higher self-renewal ability and chemoresistance. CD271 expression was associated with TNM stage and metastatic capacity in human ESCC and we found that CD271 expression was regulated by DNA methylation. Our results showed that CD271 ${ }^{+}$ESCC cells possess stem-like properties and their expression is epigenetically regulated.

\section{Materials and methods}

Cell line and tissue specimens. The KYSE70 ESCC cell line was preserved in our laboratory and maintained in RMPI-1640 supplemented with 10\% fetal bovine serum (both from Hyclone, Logan, UT, USA), $100 \mathrm{U} / \mathrm{ml}$ of penicillin, and $100 \mu \mathrm{g} / \mathrm{ml}$ of streptomycin at $37^{\circ} \mathrm{C}, 5 \% \mathrm{CO}_{2}$. Sixty-three paired ESCC tissues and adjacent non-cancerous tissues were previously collected and stored (2008-2010). Tissues were provided by the Department of Pathology, The First Affiliated Hospital of Zhengzhou University, with confirmed histopathological results. Information pertaining to clinicopathological parameters were also available.

Flow cytometric analysis and cell sorting. Adherent KYSE70 cells were trypsinized and dissociated into single cells suspended in PBS with 3\% fetal bovine serum. The cells were stained with PE-conjugated mouse anti-human CD271 monoclonal antibody (BD Biosciences, San Jose, CA, USA). The corresponding isotype immunoglobulins were used as controls. Dead cells were identified using 7-AAD (Biolegend, San Diego, CA, USA). Samples were analyzed using BD FACS Canto II cytometer (BD Biosciences) and sorted with a MoFlo XDP cytometer (Beckman, Brea, CA, USA).

RNA extraction and cDNA synthesis. Total RNA was extracted from KYSE70 cells and tissue specimens by TRIzol reagent (Invitrogen Life Technologies, Carlsbad, CA, USA) according to the manufacturer's instructions. The first-strand cDNA was synthesized from $1 \mu \mathrm{g}$ of total RNA using PrimeScript RT reagent kit with gDNA Eraser (Takara, Shiga, Japan).

Quantitative real-time PCR. The cDNA was used as a template to detect the expression of CD271 in KYSE70 cells and tissue specimens. qPCR was performed using SYBR Premix Ex Taq II (Takara) and assessed by Agilent Mx3005P. GAPDH was used as an internal control. The data were analyzed by $2^{-\Delta \Delta C \mathrm{t}}$. Primer sequences for qPCR are shown in Table I.

Sphere formation assay. The sorted CD271 ${ }^{+}$and CD271- cells were resuspended in DMEM/F12 medium (Invitrogen Life Technologies) supplemented with $4 \mu \mathrm{g} / \mathrm{ml}$ heparin (Sigma, St. Louis, MO, USA), B27 (1:50, Gibco, Life Technologies, Carlsbad, CA, USA), 20 ng/ml EGF, 20 ng/ml basic FGF (both from PeproTech, Rocky Hill, NJ, USA), penicillin 100 IU/ $\mathrm{ml}$ and streptomycin $100 \mu \mathrm{g} / \mathrm{ml}$ and then seeded in 24-well ultra-low cluster plates (Corning Costar, Corning, NY, USA).
After culturing for 7 days, the number of spheres was counted under a microscope (Leica, Wetzlar, Germany).

Anchorage-independent cell growth. The sorted CD $271^{+}$and CD271- cells $\left(2 \times 10^{3}\right.$ cells/well) were suspended in complete medium containing $0.6 \%$ low-melting-point agar (Sigma) and then applied to the top of a $1.2 \%$ agar/complete medium layer in six-well plates. After 14 days, surviving colonies were stained with $0.4 \%$ crystal violet (Sigma) and counted under a microscope (Leica, USA). The experiments were carried out in triplicate wells. Representative images were taken.

Drug sensitivity assay and chemotherapy resistance assay. 5-Fluorouracil (5-FU) and Cisplatin (DDP), both from Sigma, were dissolved according to the manufacturer's instructions. The sorted CD $271^{+}$and CD $271^{-}$cells were seeded at $3 \times 10^{3}$ cells/well in 96-well plates and treated with chemotherapeutic reagents in quadruplicate. Cell viability was evaluated using CCK-8 assay (Biyuntian, Jiangsu, China) following treatment with chemotherapeutic reagents for $48 \mathrm{~h}$, and the absorbance was measured at $450 \mathrm{~nm}$ using Multiskan Mk3 (Thermo Fisher Scientific, San Jose, CA, USA). The percentage of survival in treated cells was normalized with untreated controls.

KYSE70 cells were continuously treated with DDP $(0.5 \mu \mathrm{M})$ and 5-FU $(0.5 \mu \mathrm{g} / \mathrm{ml})$ until significant DDP and 5-FU resistance in KYSE70 cells was observed via cell viability assessment. Flow cytometry was then used to detect the CD271 expression.

Bisulfite modification and methylation analysis. Genomic DNA extracted from the KYSE70 cell line using the Takara MiniBEST Universal Genomic DNA Extraction kit (Takara), was modified by sodium bisulfite using the EpiTect Bisulfite kit (Qiagen, Germany) according to the manufacturer's instructions. Methylation status was analyzed by bisulfite genomic sequencing (BSP) of the $\mathrm{CpG}$ islands. The region was amplified using the primers shown in Table I. Amplified products were cloned into pMD-18T simple vector (Takara), transformed into DH5 $\alpha$ competent cells (Takara), and plated under ampicilin selection. Five independent clones were sequenced.

Statistical analysis. Data were expressed as mean \pm SD and analyzed using the Student's t-test. Paired t-test was used for paired samples. Non-parametric test was performed for samples of non-normal distribution. Statistical analyses were conducted with SPSS 17.0 software. $\mathrm{P}<0.05$ was considered to indicate a statistically significant difference.

\section{Results}

CD271 expression is associated with stage and lymph node metastasis in human ESCC specimens. CD271 is considered crucial to maintain tumorigenecity and stem-like properties of cancer cells including melanoma, hypopharyngeal cancer and esophageal cancer. We compared the expression of CD271 in paired human ESCC specimens and adjacent non-cancerous tissues. As shown in Fig. 1A, qPCR analysis revealed frequent upregulation of CD271 mRNA expression in carcinoma tissues compared with adjacent non-cancerous tissues, indicating that CD271 may act as an oncogene in human ESCC. Moreover, 
Table I. Primer sequences for qPCR for all the genes tested.

\begin{tabular}{|c|c|c|}
\hline Gene name & Sequence & Product size (bp) \\
\hline \multicolumn{3}{|l|}{ qPCR } \\
\hline GAPDH-F & GCACCGTCAAGGCTGAGAAC & 138 \\
\hline GAPDH-R & TGGTGAAGACGCCAGTGGA & \\
\hline CD271-F & AACAAGACCTCATAGCCAGCA & 119 \\
\hline CD271-R & CAGGATGGAGCAATAGACAGG & \\
\hline NANOG-F & CAAAGGCAAACAACCCACTT & 158 \\
\hline NANOG-R & TCTGCTGGAGGCTGAGGTAT & \\
\hline FIBRO-F & CAGTGGGAGACCTCGAGAAGA & 169 \\
\hline FIBRO-R & GTCCCTCGGAACATCAGAAAC & \\
\hline VIM-F & GAGAACTTTGCCGTTGAAGC & 163 \\
\hline VIM-R & GCTTCCTGTAGGTGGCAATC & \\
\hline BAK-F & CATCAACCGACGCTATGACTC & 192 \\
\hline BAK-R & GTCAGGCCATGCTGGTAGAC & \\
\hline CASP3-F & AGAACTGGACTGTGGCATTGAG & 191 \\
\hline CASP3-R & GCTTGTCGGCATACTGTTTCAG & \\
\hline CASP9-F & CTCAGACCAGAGATTCGCAAAC & 116 \\
\hline CASP9-R & GCATTTCСССТСАААСТСТСАА & \\
\hline \multicolumn{3}{|l|}{ BSP } \\
\hline CD271-BF & GAGAGAGAGAGGGTTGAAATTAG & 505 \\
\hline CD271-BR & AАACATССТАААТТССААСААА & \\
\hline
\end{tabular}

qPCR, quantitative polymerase chain reaction; BSP, bisulfite genomic sequencing.
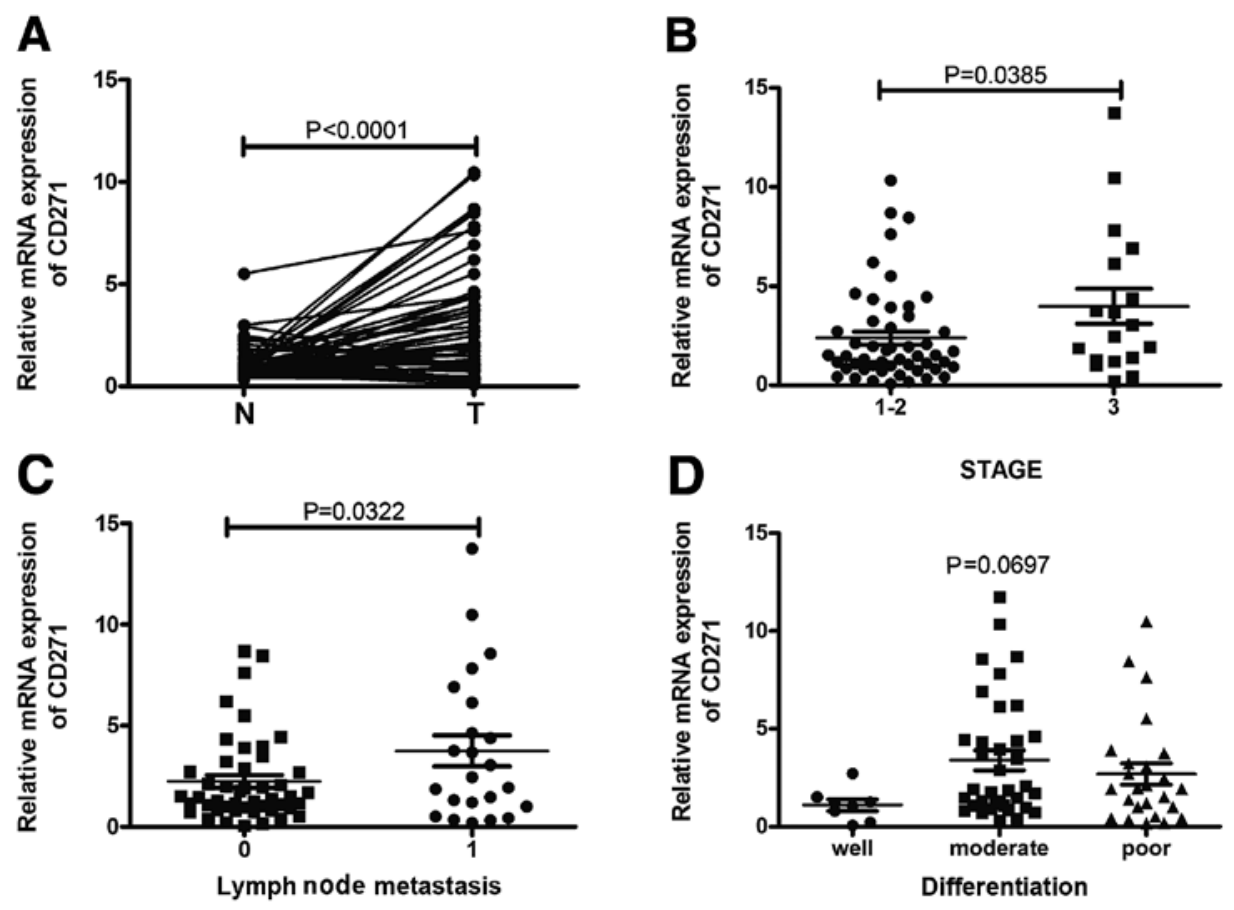

Figure 1. The expression of CD271 is associated with stage and lymph node metastasis in human ESCC specimens. (A) The mRNA expression of CD271 was investigated in paired human ESCC specimens (T) and adjacent non-cancerous tissues $(\mathrm{N})$ by qPCR. The correlation of CD271 expression with clinicopathological characteristics such as (B) stage, (C) lymph node metastasis and (D) differentiation was analyzed.

the expression of CD271 was significantly correlated with TNM stage and lymph node metastasis but not other variables such as age, gender and differentiation (Fig. 1B-D).
$C D 271^{+}$cells overexpress stem-related gene NANOG and EMT markers. To study the stem cell-like properties of isolated $\mathrm{CD} 271^{+}$cells from the KYSE70 ESCC cell line, we first detected 
A
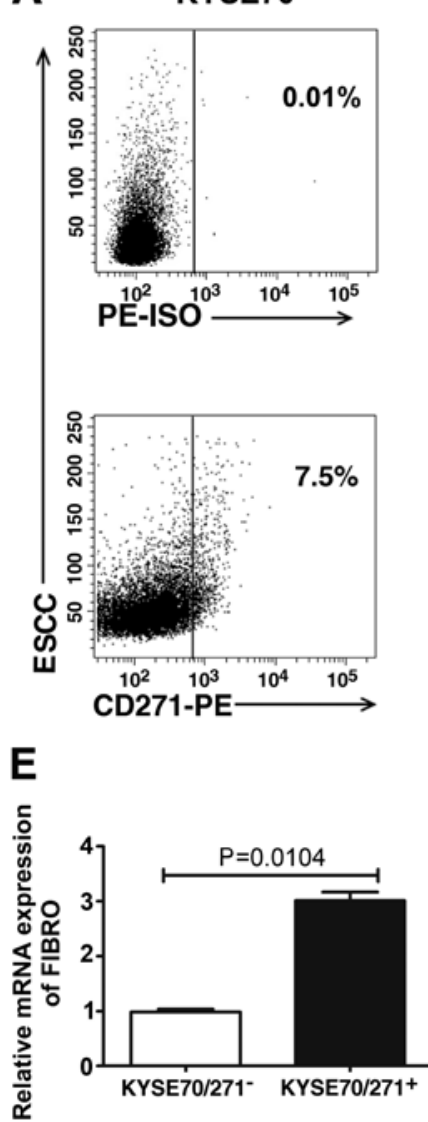

B
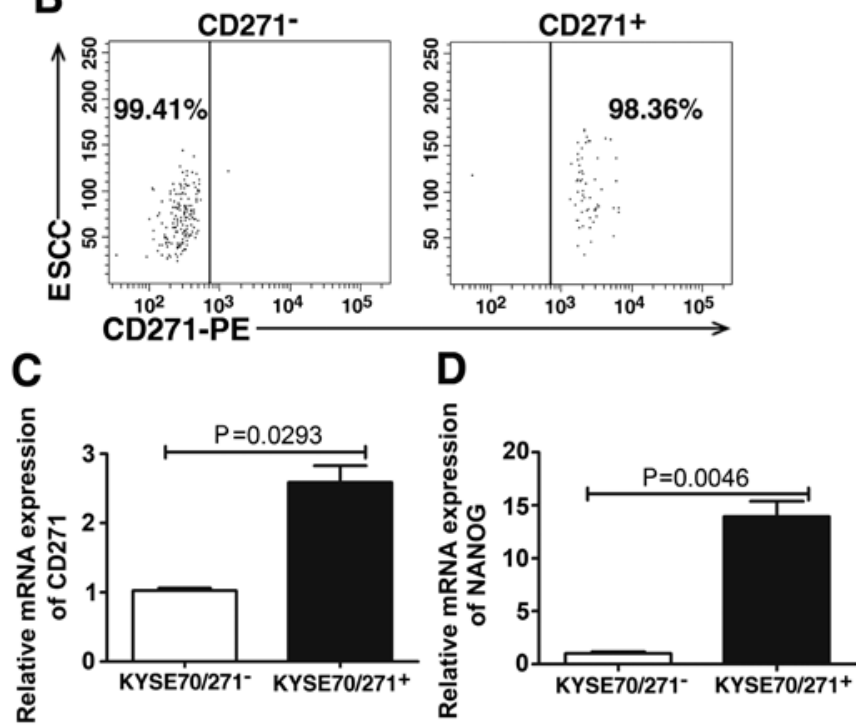

$\mathbf{F}$
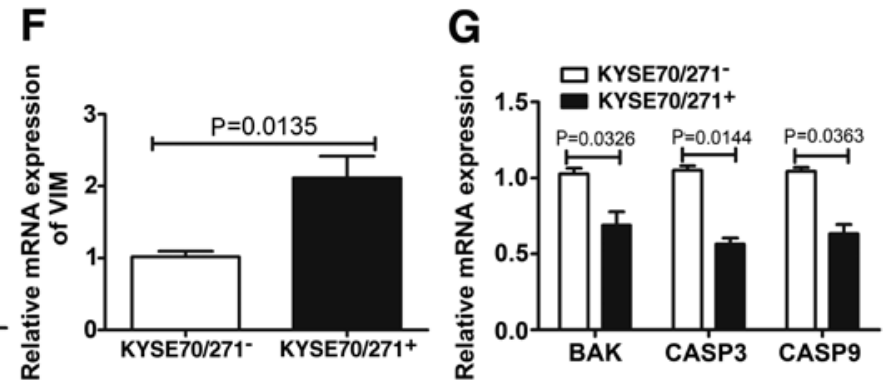

Figure 2. CD271 ${ }^{+}$cells express high levels of stem-related gene NANOG and EMT markers. (A) CD271 expression in KYSE70 was detected by flow cytometry. Of the cells 7.5\% intensely expressed CD271. (B) The purity of sorted CD271 ${ }^{+}$and CD271 cells was 99.41 and $98.36 \%$, respectively. (C) Quantitative real-time PCR analysis of CD271 expression in sorted CD271 ${ }^{+}$and CD271 cells. In the two subpopulations, relative expression of (D) NANOG, (E) Fibronectin (FIBRO), (F) Vimentin (VIM) and (G) apoptosis genes BAK, Caspase 3 and Caspase 9 was analyzed by qPCR.

the CD271 expression by flow cytometric analysis. We found the $\mathrm{CD} 271^{+}$subpopulation was $7.5 \%$ present in the KYSE70 cell line (Fig. 2A). CD271 ${ }^{+}$and CD271 ${ }^{-}$cells were then sorted separately and the purity of the two sorted subpopulations was 98.36 and $99.41 \%$, respectively (Fig. 2B). qPCR was used to confirm the expression of CD271 in the two subpopulations (Fig. 2C). We also compared the expression of the stem-related gene NANOG, EMT markers Fibronectin (FIBRO) and Vimentin (VIM), and apoptosis genes BAK, Caspase 3 and Caspase 9 in $\mathrm{CD}_{271^{+}}$and CD271- cells. Our results showed that compared with $\mathrm{CD} 271^{-}$cells, $\mathrm{CD} 271^{+}$cells exhibited an increased expression of NANOG, FIBRO, VIM (Fig. 2D-F) and a decreased expression of BAK, Caspase 3 and Caspase 9 (Fig. 2G).

CD271+ cells form more self-renewing spheres and promote anchorage-independent growth. One of the most important properties of GSCs is self-renewal. We examined the tumor sphere formation ability, which represents a self-renewal capacity. The sorted CD $271^{+}$and CD271 ${ }^{-}$cells were seeded in 24-well ultra-low cluster plates at a density of $5 \times 10^{3}$ cells $/ \mathrm{ml}$ in DMEM/F12 medium supplemented with heparin, B27, EGF, and bFGF. After culturing for 7 days, CD271+ cells exhibited a marked ability for tumor sphere formation, compared with CD271- cells (Fig. 3A and B). In addition, CD271 ${ }^{+}$cells promoted anchorage-independent growth (Fig. 3C), indicating that the $\mathrm{CD} 271^{+}$cells play an important role in the maintenance of malignant growth of ESCC cells. These results suggested that $\mathrm{CD} 271^{+}$ESCC cells possess stem-like properties.

$C D 271^{+}$cells possess the ability to resist conventional chemotherapeutic reagents in vitro. Cancer stem cells are more resistant to conventional chemotherapeutic drugs. To examine whether the self-renewing $\mathrm{CD} 271^{+}$cells possess the hypothesized CSC chemoresistant ability, the sensitivity of the sorted CD271 ${ }^{+}$and CD271 ${ }^{-}$cells to DDP and 5-FU, respectively, was analyzed. The survival rates of $\mathrm{CD} 271^{+}$cells were higher under the treatment of DDP and 5-FU, compared with CD271' cells (Fig. 4A and B). We also identified CD $271^{+}$stem-like cells could be enriched in DDP- and 5-FU-resistant cells (Fig. 4C-G). The results validated a role for $\mathrm{CD} 271^{+}$cells in chemoresistance, which may explain the failure of current therapies to eradicate progenitors and prevent tumor recurrence.

CD271 expression is regulated by DNA methylation. Transcription factors and epigenetic modifications often guide external signals to a specific genetic response. We examined whether epigenetics including DNA methylation are involved in regulating CD271 expression. To investigate promoter methylation of CD271, BSP was performed. The area of the $\mathrm{CpG}$-rich region spanning $31 \mathrm{CpG}$ sites, was sequenced (Fig. 5A). We found most $\mathrm{CpG}$ dinucleotides were methylated 
A

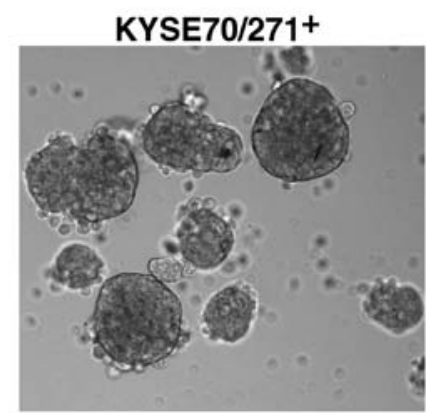

B

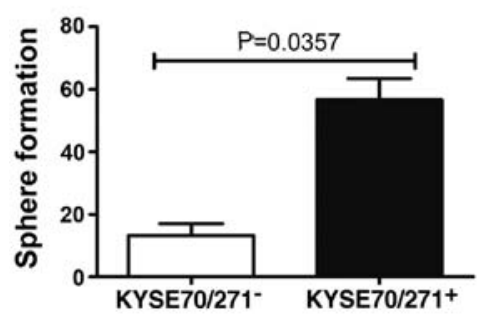

KYSE70/271-

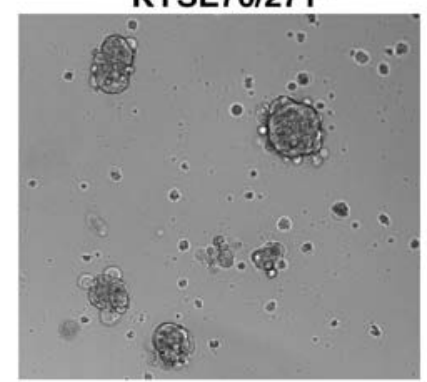

C

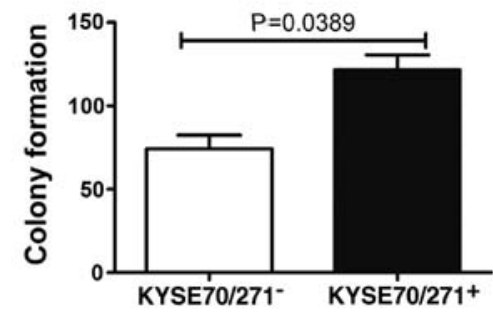

Figure 3. CD271 cells form more self-renewing spheres and promote anchorage-independent growth. (A and B) The capability of tumor sphere formation was measured in sorted CD271 ${ }^{+}$and CD271- cells. (C) Anchorage-independent soft-agar growth was analyzed in sorted CD271 ${ }^{+}$and CD271- cells.
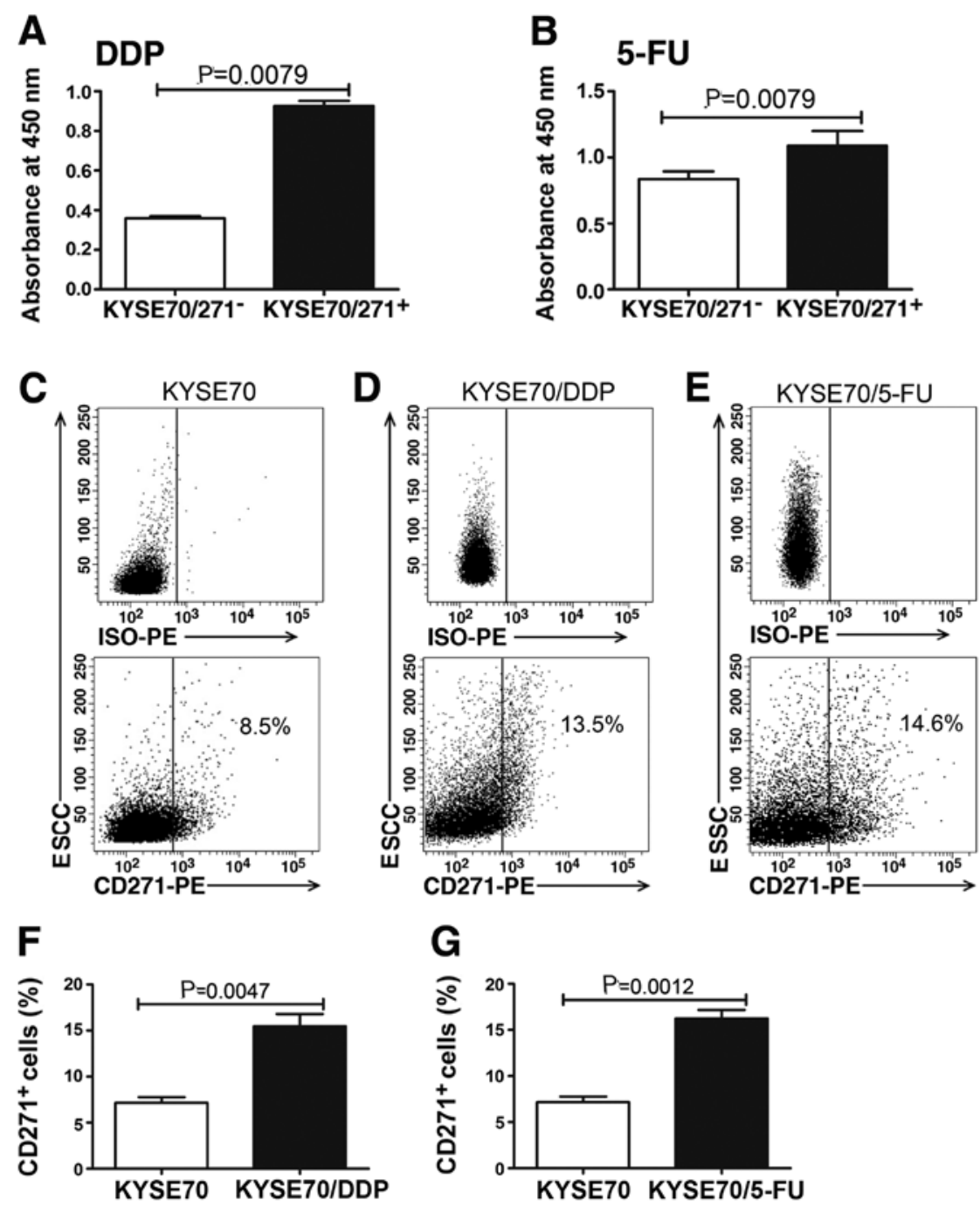

Figure $4 . \mathrm{CD}_{271^{+}}$cells possess the ability to resist to conventional chemotherapeutic reagents in vitro. Surviving cells were detected in sorted CD271 ${ }^{+}$and CD271- cells treated with (A) DDP and (B) 5-FU by CCK8 assay. CD271 expression was analyzed in (D and F) DDP-, (E and G) 5-FU-resistant cells and (C) parent cells by flow cytometry. 


\begin{abstract}
A
GAGAGAGAGAGGGCTGAAACCAGAGCGCGTCCCGGCGGGTCGGCTGGCGGGCCGCGCCGGTAATGGAGGCACTTT GTCATTCAGACGTCTGTAACCAGAGCCGCCGGGCTGGCTAATGCGCCTAATAGGGATGGAACGAGGGCAGCAAATGG GCGTGCGTGAGCGGCCGGGCTGAGCTGGGTGGATGGTGGATGGGGAGGCAGCTCCGCGGGGGACGGGCCCCTAC CGCACTGTTCCAGCCCAGTCTGGCTCAAGCGCCTCGCTTCTTCCCTGGGGGACCGCGTGGGGAGGCGGAATAATCG CATGTGCTGAGTTCCTACTAAGTGCCTTGCACTTCCCTGGTAGTTTCTCTTGAATTATTTAATCCTTATAAGGTCCTAGCA AGGTGAATTTAGTTATTCCCACTTCGCAGTTGAGGAAACAGAACCTTCTAGACTTTCAGGCCCGAGACCAGGCTCCCCC AGTGAGTCAGTGGTGGCGTCTTTGTTGGAACCCAGGATGCTT
\end{abstract}

$\mathrm{B}$

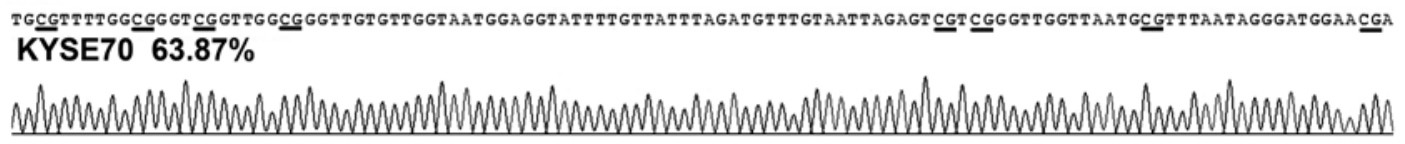

C TGTGTTTTGGTGGTTGGTTGGTGGTTGTGTCGGTAATGGGGTATTTTGTTATTAGATGTTTGTAATAGAGTTGTGGGTTGGTAATGTGTTPAATAGGATGGAATGA KYSE70+5-aza $\quad 32.90 \%$

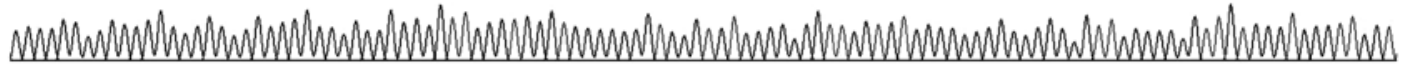

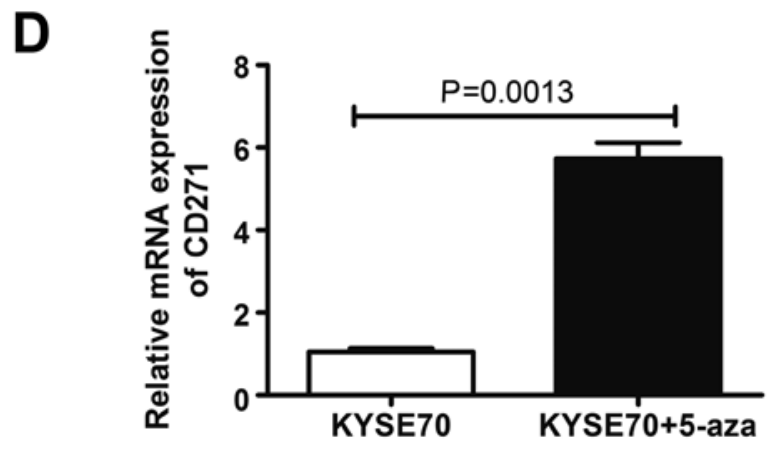

Figure 5. CD271 expression is regulated by DNA methylation. (A) CD271 promoter region. The analyzed CpG dinucleotides are underlined. Primers used for bisufilte genomic sequencing are in bold. Methylation status of $31 \mathrm{CpG}$ sites in the promoter region of KYSE70 cells treated (B) without or (C) with demethylation agent 5-aza. (D) qPCR analysis of CD271 expression in KYSE70 cells treated with or without 5-aza.

in KYSE70 cells (Fig. 5B). To elucidate whether the methylation of CD271 was associated with its expression, KYSE70 cells were treated with 5-aza (an inhibitor of the methylase enzyme, which can reactivate mRNA expression suppressed by methylation) for 6 days and performed BSP and qPCR to detect the promoter methylation and expression of CD271. Following treatment with $5 \mu \mathrm{M} 5$-aza for 6 days, CD271 gene exhibited an obvious induction and the promoter methylation level was reduced (Fig. 5C and D), suggesting that the expression of CD271 is regulated by DNA methylation.

\section{Discussion}

The majority of ESCC patients present with an advanced stage at the time of diagnosis, with poor prognosis, rapid growth and spread due to late diagnosis. Accumulating evidence suggested the CSCs theory in that tumorigenic potential is largely restricted to CSCs $(21,22)$. CSCs from tumor tissues or established cancer cell lines can be isolated by cell-surface markers expressed on CSCs $(17,23)$. In recent studies ALDH was identified as the CSC marker for various types of cancer including ovarian (24), breast (25), lung (26) and prostate (27) cancer, $\mathrm{CD} 44^{+} \mathrm{CD} 24^{-}$for breast (28) and ovarian (29) cancer, CD133 for non-small cell lung (30), liver (31) and lung (32) cancer. However, Meng et al reported that both $\mathrm{CD}_{133^{+}}$and CD133- subpopulations contain similar numbers of CSCs (33). Therefore, identification of specific cell-surface markers to define CSCs is important for the possible establishment of target-specific therapies using small molecule inhibitors or humanized antibodies.

CD271 knockdown was found to eliminate the capacity of melanoma cells to form heterogeneous tumors most likely through the downregulation of mediators for melanoma invasion and metastasis (GLI-2, SOX2 and ERBB3), angiogenesis (IGFBP-2), proliferation (FST and MITF) or chemoresistance (RHOJ) (34). CD271 was also identified as a predominant molecule responsible for the proliferation, tumorigenecity and plasticity of melanoma cells. Accumulating evidence has shown that metastases develop when distant organs are seeded with CSCs derived from a primary tumor (35). Boiko et al identified that $\mathrm{CD} 271^{+}$melanoma cells lacked the expression of TYR, MART1 and MAGE genes in 86, 69 and 68\% of melanoma patients, respectively, which may explain the reason for T-cell 
therapies targeting these antigens usually resulting in only temporary tumor shrinkage (16). Okumura et al have shown that CD271 expression correlated with negative lymph node metastasis and lower TNM staging. There was no significant correlation between CD271 expression and distant metastasis in ESCC (19). However, in this study we show that the expression of CD271 was significantly higher in ESCC tissues than adjacent non-cancerous tissues. In contrast to findings of a previous study (19), we found that the overexpression of CD271 was significantly associated with TNM stage and lymph node metastasis. Taken together, these data strongly suggest CD271 as an oncogene that plays an important role in ESCC progression.

Cell sorting yielded CD271 ${ }^{+}$and $\mathrm{CD} 271^{-}$cells and identified the overexpression of the stem-related gene NANOG in $\mathrm{CD} 271^{+}$cells. Since EMT plays a key role in tumor invasion and metastasis during tumor progression, we tested the expression of EMT markers between the two subpopulations. As expected, CD $271^{+}$cells highly expressed mesenchymal markers, such as FIBRO and VIM. We also found that CD271 ${ }^{+}$ cells were able to form more tumor spheres and colonies compared with CD271 ${ }^{-}$cells. Our results further showed that CD271 $1^{+}$cells exhibited general resistance to DDP and 5-FU, with higher survival percentages compared with CD271 ${ }^{-}$cells. Taken together, these data suggest that CD271 is a marker of stem-like cells in ESCC. In addition, we observed increased $\mathrm{CD} 271^{+}$cells in DDP- and 5-FU-resistant cells. In other words, conventional chemotherapeutic drugs, such as DDP and 5-FU, may selectively decrease the CD271- population, resulting in a relative increase of $\mathrm{CD} 271^{+}$cell frequency. Thus, CSCs can be responsible for treatment failures of chemotherapy and poor clinical outcomes.

Accumulating evidence has suggested that CSCs are not only governed by genetic alterations but also aberrant epigenetic regulation. Sun et al reported that a unique set of genes such as NANOG, OCT4 and SOX9, were demethylated in invasive cancer cells but methylated in non-invasive cancer cells, indicating that they may be biologically important in the invasive population and upregulated during the EMT process (36). Schirmer et al (39) found that the gene L1CAM, which can promote cell motility, invasion and metastasis formation in various human cancers (37-39), could be induced by treatment with the demethylation agent 5-aza in endometrial carcinoma cell lines (40). We previously found that tumor-suppressor gene SPINT2 was induced by a demethylation agent (41). This shows that following epigenetic drug treatment, hypermethylated tumor-suppressor genes as well as oncogenes may be activated simultaneously. In the present study, we firstly found CD271 was regulated epigenetically and its expression could be induced by demethylation agent treatment. Therefore, epigenetic therapy may be a 'double-edge sword' and unexpected side effects may occur.

In conclusion, we have demonstrated that CD271, the ESCC cancer stem cell marker, is a potential prognostic marker of patients with ESCC and is regulated epigenetically.

\section{Acknowledgements}

This study was supported by grants from the China-US (NFSC-NIH) Program for Biomedical Collaborative
Research (Grant no. 812111102), the National Natural Science Foundation of China (Grant no. 81171986), Research Grant from the Ministry of Public Health (no. 20110110001), the Basic and Advanced Technology Research Foundation from Science and Technology Department of Henan Province (Grant no. 112300410153, Grant no. 122300410155), Funds for Creative Research Team of Henan Province, Creative Research Team of Higher Education of Henan Province and the Innovation Team of The First Affiliated Hospital of Zhengzhou University.

\section{References}

1. Yuequan J, Shifeng C and Bing Z: Prognostic factors and family history for survival of esophageal squamous cell carcinoma patients after surgery. Ann Thorac Surg 90: 908-913, 2010.

2. Reya T, Morrison SJ, Clarke MF and Weissman IL: Stem cells, cancer, and cancer stem cells. Nature 414: 105-111, 2001.

3. Boman BM and Wicha MS: Cancer stem cells: a step toward the cure. J Clin Oncol 26: 2795-2799, 2008.

4. Wicha MS, Liu S and Dontu G: Cancer stem cells: an old idea - a paradigm shift. Cancer Res 66: 1883-1890, 2006.

5. Hermann PC, Huber SL, Herrler T, et al: Distinct populations of cancer stem cells determine tumor growth and metastatic activity in human pancreatic cancer. Cell Stem Cell 1: 313-323, 2007.

6. Frank NY, Margaryan A, Huang Y, et al: ABCB5-mediated doxorubicin transport and chemoresistance in human malignant melanoma. Cancer Res 65: 4320-4333, 2005.

7. Zhang Y, Wang Z, Yu J, et al: Cancer stem-like cells contribute to cisplatin resistance and progression in bladder cancer. Cancer Lett 322: 70-77, 2012.

8. Cho RW and Clarke MF: Recent advances in cancer stem cells. Curr Opin Genet Dev 18: 48-53, 2008.

9. Tilghman J, Wu H, Sang Y, et al: HMMR maintains the stemness and tumorigenicity of glioblastoma stem-like cells. Cancer Res 74: 3168-3179, 2014.

10. Hirsch D, Barker N, McNeil N, et al: LGR5 positivity defines stem-like cells in colorectal cancer. Carcinogenesis 35: 849-858, 2014.

11. Ding K, Banerjee A, Tan S, et al: Artemin, a member of the glial cell line-derived neurotrophic factor family of ligands, is HER2 regulated and mediates acquired Trastuzumab resistance by promoting cancer stem cell-like behaviour in mammary carcinoma cells. J Biol Chem 289: 16057-16071, 2014.

12. Liepinsh E, Ilag LL, Otting G and Ibanez CF: NMR structure of the death domain of the p75 neurotrophin receptor. EMBO J 16: 4999-5005, 1997.

13. Rabizadeh S, Oh J, Zhong LT, et al: Induction of apoptosis by the low-affinity NGF receptor. Science 261: 345-348, 1993.

14. Bibel $M$ and Barde YA: Neurotrophins: key regulators of cell fate and cell shape in the vertebrate nervous system. Genes Dev 14: 2919-2937, 2000.

15. Civenni G, Walter A, Kobert N, et al: Human CD271-positive melanoma stem cells associated with metastasis establish tumor heterogeneity and long-term growth. Cancer Res 71: 3098-3109, 2011.

16. Boiko AD, Razorenova OV, van de Rijn M, et al: Human melanoma-initiating cells express neural crest nerve growth factor receptor CD271. Nature 466: 133-137, 2010.

17. Imai T, Tamai K, Oizumi S, et al: CD271 defines a stem cell-like population in hypopharyngeal cancer. PLoS One 8: e62002, 2013.

18. Okumura T, Shimada Y, Imamura M and Yasumoto S: Neurotrophin receptor $\mathrm{p} 75$ (NTR) characterizes human esophageal keratinocyte stem cells in vitro. Oncogene 22: 4017-4026, 2003.

19. Okumura T, Tsunoda S, Mori Y, et al: The biological role of the low-affinity p75 neurotrophin receptor in esophageal squamous cell carcinoma. Clin Cancer Res 12: 5096-5103, 2006.

20. Huang SD, Yuan Y, Liu XH, et al: Self-renewal and chemotherapy resistance of p75NTR positive cells in esophageal squamous cell carcinomas. BMC Cancer 9: 9, 2009.

21. Clarke MF, Dick JE, Dirks PB, et al: Cancer stem cells perspectives on current status and future directions: AACR Workshop on cancer stem cells. Cancer Res 66: 9339-9344, 2006.

22. Eyler CE and Rich JN: Survival of the fittest: cancer stem cells in therapeutic resistance and angiogenesis. J Clin Oncol 26: 2839-2845, 2008. 
23. Tirino V, Desiderio V, d'Aquino R, et al: Detection and characterization of $\mathrm{CD}_{133^{+}}$cancer stem cells in human solid tumours PLoS One 3: e3469, 2008.

24. Wang YC, Yo YT, Lee HY, et al: ALDH1-bright epithelia ovarian cancer cells are associated with CD44 expression, drug resistance, and poor clinical outcome. Am J Pathol 180: $1159-1169,2012$

25. Marcato P, Dean CA, Pan D, et al: Aldehyde dehydrogenase activity of breast cancer stem cells is primarily due to isoform ALDH1 $\mathrm{A} 3$ and its expression is predictive of metastasis. Stem Cells 29: 32-45, 2011.

26. Jiang F, Qiu Q, Khanna A, et al: Aldehyde dehydrogenase 1 is a tumor stem cell-associated marker in lung cancer. Mol Cancer Res 7: 330-338, 2009.

27. Hellsten R, Johansson M, Dahlman A, Sterner O and Bjartell A: Galiellalactone inhibits stem cell-like ALDH-positive prostate cancer cells. PLoS One 6: e22118, 2011.

28. Sun H, Jia J, Wang X, et al: CD44(+)/CD24(-) breast cancer cells isolated from MCF-7 cultures exhibit enhanced angiogenic properties. Clin Transl Oncol 15: 46-54, 2012

29. Meng E, Long B, Sullivan P, et al: $\mathrm{CD}_{4} 4^{+} / \mathrm{CD} 24$ ovarian cancer cells demonstrate cancer stem cell properties and correlate to survival. Clin Exp Metastasis 29: 939-948, 2012.

30. Tirino V, Camerlingo R, Franco R, et al: The role of CD133 in the identification and characterisation of tumour-initiating cells in non-small-cell lung cancer. Eur J Cardiothorac Surg 36: 446-453, 2009.

31. Piao LS, Hur W, Kim TK, et al: CD133+ liver cancer stem cells modulate radioresistance in human hepatocellular carcinoma. Cancer Lett 315: 129-137, 2012.

32. Bertolini G, Roz L, Perego P, et al: Highly tumorigenic lung cancer $\mathrm{CD} 133^{+}$cells display stem-like features and are spared by cisplatin treatment. Proc Natl Acad Sci USA 106: 16281-16286, 2009.
33. Meng X, Li M, Wang X, Wang Y and Ma D: Both CD133+ and CD133- subpopulations of A549 and H446 cells contain cancer-initiating cells. Cancer Sci 100: 1040-1046, 2009.

34. Redmer T, Welte Y, Behrens D, et al: The nerve growth factor receptor CD271 is crucial to maintain tumorigenicity and stem-like properties of melanoma cells. PLoS One 9: e92596, 2014.

35. Balic M, Lin H, Young L, et al: Most early disseminated cancer cells detected in bone marrow of breast cancer patients have a putative breast cancer stem cell phenotype. Clin Cancer Res 12: 5615-5621, 2006

36. Sun L, Mathews LA, Cabarcas SM, et al: Epigenetic regulation of SOX9 by the NF- $\kappa \mathrm{B}$ signaling pathway in pancreatic cancer stem cells. Stem Cells 31: 1454-1466, 2013.

37. Chen DL, Zeng ZL, Yang J, et al: L1CAM promotes tumor progression and metastasis and is an independent unfavorable prognostic factor in gastric cancer. J Hematol Oncol 6: 43, 2013.

38. Ben Q, An W, Fei J, et al: Downregulation of L1CAM inhibits proliferation, invasion and arrests cell cycle progression in pancreatic cancer cells. Exp Ther Med 7: 785-790, 2014.

39. Schirmer U, Doberstein K, Rupp AK, et al: Role of miR-34a as a suppressor of L1CAM in endometrial carcinoma. Oncotarget 5: 462-472, 2014

40. Schirmer U, Fiegl H, Pfeifer M, et al: Epigenetic regulation of L1CAM in endometrial carcinoma: comparison to cancer-testis (CT-X) antigens. BMC Cancer 13: 156, 2013.

41. Yue D, Fan Q, Chen X, et al: Epigenetic inactivation of SPINT2 is associated with tumor suppressive function in esophageal squamous cell carcinoma. Exp Cell Res 322: 149-158, 2014. 